\title{
Existence and uniqueness of solutions for a class of integral equations by common fixed point theorems in IFMT-spaces
}

\section{Reza Saadati*}

"Correspondence: rsaadati@eml.cc Department of Mathematics, Iran

University of Science and

Technology, Tehran, Iran

\begin{abstract}
In this paper, our aim is to address the existence and uniqueness of solutions for a class of integral equations in IFMT-space. Therefore, we introduce the concept of IFMT-spaces and prove a common fixed point theorem in a complete IFMT-space; next we study an application.
\end{abstract}

MSC: 54E40; 54E35; 54H 25

Keywords: integral equations; nonlinear IF contractive mapping; complete IFMT-space; fixed point theorem

\section{Introduction and preliminaries}

First of all, we would like to introduce the concept of IFMT-space, which is a non-trivial generalization of IFM-space introduced by Park [1] and Saadati and Park [2] and Saadati et al. [3]; also we use results from [4-8].

We say the pair $\left(L^{*}, L_{L^{*}}\right)$ is a complete lattice whenever $L^{*}$ is a non-empty set and we have the operation $\leq_{L^{*}}$ defined by

$$
L^{*}=\{(a, b):(a, b) \in[0,1] \times[0,1] \text { and } a+b \leq 1\},
$$

$(a, b) \leq_{L^{*}}(c, d) \Longleftrightarrow a \leq c$, and $b \geq d$, for each $(a, b),(c, d) \in L^{*}$

Definition 1.1 ([9]) An IF set $\mathcal{F}_{\alpha, \beta}$ in a universe $U$ is an object $\mathcal{F}_{\alpha, \beta}=\left\{\left(\alpha_{\mathcal{F}}(u), \beta_{\mathcal{F}}(u)\right) \mid u \in\right.$ $U\}$, in which, for all $u \in U, \alpha_{\mathcal{F}}(u) \in[0,1]$, and $\beta_{\mathcal{F}}(u) \in[0,1]$ are said the membership degree and the non-membership degree, respectively, of $u$ in $\mathcal{F}_{\alpha, \beta}$, and furthermore they satisfy $\alpha_{\mathcal{F}}(u)+\beta_{\mathcal{F}}(u) \leq 1$.

We consider $0_{L^{*}}=(0,1)$ and $1_{L^{*}}=(1,0)$ as its units.

Definition $1.2([4])$ The mapping $\mathcal{T}: L^{*} \times L^{*} \longrightarrow L^{*}$ satisfying the following conditions:

$$
\begin{aligned}
& \left(\forall a \in L^{*}\right)\left(\mathcal{T}\left(a, 1_{L^{*}}\right)=a\right), \\
& \left(\forall(a, b) \in L^{*} \times L^{*}\right)(\mathcal{T}(a, b)=\mathcal{T}(b, a)), \\
& \left(\forall(a, b, c) \in L^{*} \times L^{*} \times L^{*}\right)(\mathcal{T}(a, \mathcal{T}(b, c))=\mathcal{T}(\mathcal{T}(a, b), c)), \\
& \left(\forall\left(a, a^{\prime}, b, b^{\prime}\right) \in L^{*} \times L^{*} \times L^{*} \times L^{*}\right)\left(a \leq_{L^{*}} a^{\prime} \text { and } b \leq_{L^{*}} b^{\prime} \Longrightarrow \mathcal{T}(a, b) \leq_{L^{*}} \mathcal{T}\left(a^{\prime}, b^{\prime}\right)\right) .
\end{aligned}
$$
is said to be a triangular norm $\left(t\right.$-norm) on $L^{*}$.

(c) 2016 Saadati. This article is distributed under the terms of the Creative Commons Attribution 4.0 International License (http://creativecommons.org/licenses/by/4.0/), which permits unrestricted use, distribution, and reproduction in any medium, provided you give appropriate credit to the original author(s) and the source, provide a link to the Creative Commons license, and indicate if changes were made. 
$\mathcal{T}$ is said to be a continuous $t$-norm if the triple $\left(L^{*}, \leq_{L^{*}}, \mathcal{T}\right)$ is an Abelian topological monoid with unit $1_{L^{*}}$.

Definition 1.3 ([4]) $\mathcal{T}$ on $L^{*}$ is called continuous t-representable if and only if there exist a continuous $t$-norm $*$ and a continuous $t$-conorm $\diamond$ on $[0,1]$ such that, for all $a=\left(a_{1}, a_{2}\right), b=\left(b_{1}, b_{2}\right) \in L^{*}$,

$$
\mathcal{T}(a, b)=\left(a_{1} * b_{1}, a_{2} \diamond b_{2}\right)
$$

For example, $\mathcal{T}(a, b)=\left(a_{1} b_{1}, \min \left(a_{2}+b_{2}, 1\right)\right)$ for all $a=\left(a_{1}, a_{2}\right)$ and $b=\left(b_{1}, b_{2}\right)$ in $L^{*}$ is a continuous $t$-representable.

Definition 1.4 The decreasing mapping $\mathcal{N}: L^{*} \longrightarrow L^{*}$ satisfying $\mathcal{N}\left(0_{L^{*}}\right)=1_{L^{*}}$ and $\mathcal{N}\left(1_{L^{*}}\right)=0_{L^{*}}$ is said a negator on $L^{*}$. We say $\mathcal{N}$ is an involutive negator if $\mathcal{N}(\mathcal{N}(a))=a$, for all $a \in L^{*}$. The decreasing mapping $N:[0,1] \longrightarrow[0,1]$ satisfying $N(0)=1$ and $N(1)=0$ is said to be a negator on $[0,1]$. The standard negator on $[0,1]$ is defined, for all $a \in[0,1]$, by $N_{s}(a)=1-a$, denoted by $N_{s}$. We show $\left(N_{s}(a), a\right)=\mathcal{N}_{s}(a)$.

Definition 1.5 If for given $\alpha \in(0,1)$ there is $\beta \in(0,1)$ such that

$$
\mathcal{T}^{m}\left(\mathcal{N}_{s}(\beta), \ldots, \mathcal{N}_{s}(\beta)\right)>_{L^{*}} \mathcal{N}_{s}(\alpha), \quad m \in \mathbf{N}
$$

then $\mathcal{T}$ is a $H$-type $t$-norm.

A typical example of such $t$-norms is

$$
\wedge(a, b)=\left(\operatorname{Min}\left(a_{1}, b_{1}\right), \operatorname{Max}\left(a_{2}, b_{2}\right)\right)
$$

for every $a=\left(a_{1}, a_{2}\right)$ and $b=\left(b_{1}, b_{2}\right)$ in $L^{*}$.

Definition 1.6 The tuble $\left(X, \mathcal{M}_{M, N}, \mathcal{T}\right)$ is said to be an IFMT-space if $X$ is an (non-empty) set, $\mathcal{T}$ is a continuous $t$-representable, and $\mathcal{M}_{M, N}$ is a mapping $X^{2} \times[0,+\infty) \rightarrow L^{*}$ (in which $M, N$ are fuzzy sets from $X^{2} \times[0,+\infty)$ to $[0,1]$ such that $M(x, y, t)+N(x, y, t) \leq 1$ for all $x, y \in X$ and $t>0$ ) satisfying the following conditions for every $x, y, z \in X$ and $t, s>0$ :

(a) $\mathcal{M}_{M, N}(x, y, t)>{ }_{L} 0_{L^{*}}$;

(b) $\mathcal{M}_{M, N}(x, y, t)=\mathcal{M}_{M, N}(y, x, t)=1_{L^{*}}$ iff $x=y$;

(c) $\mathcal{M}_{M, N}(x, y, t)=\mathcal{M}_{M, N}(y, x, t)$ for each $x, y \in X$;

(d) $\mathcal{M}_{M, N}(x, y, K(t+s)) \geq_{L^{*}} \mathcal{T}\left(\mathcal{M}_{M, N}(x, z, t), \mathcal{M}_{M, N}(z, y, s)\right)$ for some constant $K \geq 1$;

(e) $\mathcal{M}_{M, N}(x, y, \cdot):[0, \infty) \longrightarrow L^{*}$ is continuous.

Also $\mathcal{M}_{M, N}$ is said an IFMT. Note that for an IFMT-space

$$
\mathcal{M}_{M, N}(x, y, t)=(M(x, y, t), N(x, y, t)) .
$$

$\left(X, \mathcal{M}_{M, N}, \mathcal{T}\right)$ is called a Menger IFMT-space if

$$
\lim _{t \rightarrow \infty} \mathcal{M}_{M, N}(x, y, t)=\lim _{t \rightarrow \infty} \mathcal{M}_{M, N}(y, x, t)=1_{L^{*}} .
$$


Remark 1.7 The space of all real functions $\alpha(x), x \in[0,1]$ such that $\int_{0}^{1}|\alpha(x)|^{q} d x<\infty$, denoted by $L_{q}(0<q<1)$, is a metric type space. Consider

$$
d(\alpha, \beta)=\left(\int_{0}^{1}|\alpha(x)-\beta(x)|^{q} d x\right)^{\frac{1}{q}}
$$

for each $\alpha, \beta \in L_{q}$. Then $d$ is a metric type space with $K=2^{\frac{1}{q}}$.

Example 1.8 We consider the set of Lebesgue measurable functions on $[0,1]$ such that $\int_{0}^{1}|\alpha(x)|^{q} d x<\infty$, where $q>0$ is a real number denoted by $\mathfrak{M}$. Consider

$$
\mathcal{M}_{M, N}(x, y, t)= \begin{cases}0_{L^{*}} & \text { if } t \leq 0, \\ \left(\frac{t}{t+\left(\int_{0}^{1}|\alpha(x)-\beta(x)|^{q} d x\right)^{\frac{1}{q}}}, \frac{\left(\int_{0}^{1}|\alpha(x)-\beta(x)|^{q} d x\right)^{\frac{1}{q}}}{t+\left(\int_{0}^{1}|\alpha(x)-\beta(x)|^{q} d x\right)^{\frac{1}{q}}}\right) & \text { if } t>0 .\end{cases}
$$

So from Remark 1.7, we have $\left(M, \mathcal{M}_{M, N}, \wedge\right)$ is IFMT-space with $K=2^{\frac{1}{q}}$.

Definition 1.9 Let $\left(X, \mathcal{M}_{M, N}, \mathcal{T}\right)$ be a Menger IFMT-space.

(1) A sequence $\left\{x_{n}\right\}_{n}$ in $X$ is said to be convergent to $x$ in $X$ if, for every $\epsilon>0$ and $\lambda \in 0$, there exists a positive integer $N$ such that $\mathcal{M}_{M, N}\left(x_{n}, x, \epsilon\right)>1-\lambda$ whenever $n \geq N$.

(2) A sequence $\left\{x_{n}\right\}_{n}$ in $X$ is called a Cauchy sequence if, for every $\epsilon>0$ and $\lambda L^{*}-\left\{0_{L^{*}}\right\}$, there exists a positive integer $N$ such that $\mathcal{M}_{M, N}\left(x_{n}, x_{m}, \epsilon\right)>_{L} \mathcal{N}(\lambda)$ whenever $n, m \geq N$.

(3) A Menger IFMT-space $\left(X, \mathcal{M}_{M, N}, \mathcal{T}\right)$ is said to be complete if and only if every Cauchy sequence in $X$ is convergent to a point in $X$.

Remark 1.10 Khamsi and Kreinovich [10] proved, if $\left(X, \mathcal{M}_{M, N}, \mathcal{T}\right)$ is a IFMT-space and $\left\{u_{n}\right\}$ and $\left\{v_{n}\right\}$ are sequences such that $u_{n} \rightarrow u$ and $v_{n} \rightarrow v$, then

$$
\lim _{n \rightarrow \infty} \mathcal{M}_{M, N}\left(u_{n}, v_{n}, t\right)=\mathcal{M}_{M, N}(u, v, t)
$$

Remark 1.11 Let for each $\sigma \in L^{*}-\left\{0_{L^{*}}, 1_{L^{*}}\right\}$ there exists a $\varsigma \in L^{*}-\left\{0_{L^{*}}, 1_{L^{*}}\right\}$ (which does not depend on $n$ ) with

$$
\mathcal{T}^{n-1}(\mathcal{N}(\varsigma), \ldots, \mathcal{N}(\varsigma))>_{L} \mathcal{N}(\sigma) \text { for each } n \in\{1,2, \ldots\}
$$

Lemma 1.12 ([11]) Let $\left(X, \mathcal{M}_{M, N}, \mathcal{T}\right)$ be a Menger IFMT-space. If we define $E_{5, \mathcal{M}_{M, N}}$ : $X^{2} \longrightarrow \mathbb{R}^{+} \cup\{0\}$ by

$$
E_{\zeta, \mathcal{M}_{M, N}}(x, y)=\inf \left\{t>0: \mathcal{M}_{M, N}(x, y, t)>_{L} \mathcal{N}(\varsigma)\right\}
$$

for each $\varsigma \in L^{*}-\left\{0_{L^{*}}, 1_{L^{*}}\right\}$ and $x, y \in X$, then we have the following:

(1) For any $\sigma \in L^{*}-\left\{0_{L^{*}}, 1_{L^{*}}\right\}$, there exists a $\varsigma \in L^{*}-\left\{0_{L^{*}}, 1_{L^{*}}\right\}$ such that

$$
\begin{aligned}
& E_{\mu, \mathcal{M}_{M, N}}\left(x_{1}, x_{k}\right) \leq K E_{5, \mathcal{M}_{M, N}}\left(x_{1}, x_{2}\right)+K^{2} E_{5, \mathcal{M}_{M, N}}\left(x_{2}, x_{3}\right)+\cdots+K^{n-1} E_{5, \mathcal{M}_{M, N}}\left(x_{k-1}, x_{k}\right) \\
& \text { for any } x_{1}, \ldots, x_{k} \in X .
\end{aligned}
$$


(2) For each sequence $\left\{x_{n}\right\}$ in $X$, we have $\mathcal{M}_{M, N}\left(x_{n}, x, t\right) \longrightarrow 1_{L^{*}}$ if and only if $E_{\zeta, \mathcal{M}_{M, N}}\left(x_{n}, x\right) \rightarrow 0$. Also the sequence $\left\{x_{n}\right\}$ is Cauchy w.r.t. $\mathcal{M}_{M, N}$ if and only if it is Cauchy with $E_{\zeta, \mathcal{M}_{M, N}}$.

\section{Common fixed point theorems}

In this section we study some common fixed point theorems in Menger IFMT-spaces, ones can find similar results in others spaces at [12-19].

Definition 2.1 Let $f$ and $g$ be mappings from a Menger IFMT-space $\left(X, \mathcal{M}_{M, N}, \mathcal{T}\right)$ into itself. The mappings $f$ and $g$ are called weakly commuting if

$$
\mathcal{M}_{M, N}(f g x, g f x, t) \geq_{L} \mathcal{M}_{M, N}(f x, g x, t)
$$

for each $x$ in $X$ and $t>0$.

Now we assume that $\Phi$ is the set of all functions

$$
\phi:[0, \infty) \longrightarrow[0, \infty)
$$

which satisfy $\lim _{n \rightarrow \infty} \phi^{n}(t)=0$ for $t>0$ and are onto and strictly increasing. Also, we denote by $\phi^{n}(t)$ the $n$th iterative function of $\phi(t)$.

Remark 2.2 Note that $\phi \in \Phi$ implies that $\phi(t)<t$ for $t>0$. Consider $t_{0}>0$ with $t_{0} \leq \phi\left(t_{0}\right)$. Since $\phi$ is a nondecreasing function we get $t_{0} \leq \phi^{n}\left(t_{0}\right)$ for every $n \in\{1,2, \ldots\}$, which is a contradiction. Also $\phi(0)=0$.

Lemma 2.3 ([11]) If a Menger IFMT-space $\left(X, \mathcal{M}_{M, N}, \mathcal{T}\right)$ obeys the condition

$$
\mathcal{M}_{M, N}(x, y, t)=C, \text { for all } t>0,
$$

then we get $C=1_{L^{*}}$ and $x=y$.

Theorem 2.4 Consider the complete Menger IFMT-space $\left(X, \mathcal{M}_{M, N}, \mathcal{T}\right)$. Assume that $f$ and $g$ are weakly commuting self-mappings of $X$ such that:

(a) $f(X) \subseteq g(X)$;

(b) $f$ or $g$ is continuous;

(c) $\mathcal{M}_{M, N}(f x, f y, \phi(t)) \geq_{L} \mathcal{M}_{M, N}(g x, g y, t)$ in which $\phi \in \Phi$.

(i) Now let (1) hold and let there exist $a x_{0} \in X$ with

$$
E_{\mathcal{M}_{M, N}}\left(g x_{0}, f x_{0}\right)=\sup \left\{E_{\gamma, \mathcal{M}_{M, N}}\left(g x_{0}, f x_{0}\right): \gamma \in L^{*}-\left\{0_{L^{*}}, 1_{L^{*}}\right\}\right\}<\infty
$$

therefore $f$ and $g$ have a common fixed point which is unique.

Proof (i) Select $x_{0} \in X$ with $E_{\mathcal{M}_{M, N}}\left(g x_{0}, f x_{0}\right)<\infty$. Select $x_{1} \in X$ with $f x_{0}=g x_{1}$. Now select $x_{n+1}$ such that $f x_{n}=g x_{n+1}$. Now $\mathcal{M}_{M, N}\left(f x_{n}, f x_{n+1}, \phi^{n+1}(t)\right) \geq_{L} \mathcal{M}_{M, N}\left(g x_{n}, g x_{n+1}, \phi^{n}(t)\right)=$ $\mathcal{M}_{M, N}\left(f x_{n-1}, f x_{n}, \phi^{n}(t)\right) \geq_{L} \cdots \geq \mathcal{M}_{M, N}\left(g x_{0}, g x_{1}, t\right)$. 
We have for each $\lambda \in L^{*}-\left\{0_{L^{*}}, 1_{L^{*}}\right\}$ (see Lemma 1.9 of [11])

$$
\begin{aligned}
E_{\lambda, \mathcal{M}_{M, N}}\left(f x_{n}, f x_{n+1}\right) & =\inf \left\{\phi^{n+1}(t)>0: \mathcal{M}_{M, N}\left(f x_{n}, f x_{n+1}, \phi^{n+1}(t)\right)>_{L} \mathcal{N}(\lambda)\right\} \\
& \leq \inf \left\{\phi^{n+1}(t)>0: \mathcal{M}_{M, N}\left(g x_{0}, f x_{0}, t\right)>_{L} \mathcal{N}(\lambda)\right\} \\
& \leq \phi^{n+1}\left(\inf \left\{t>0: \mathcal{M}_{M, N}\left(g x_{0}, f x_{0}, t\right)>_{L} \mathcal{N}(\lambda)\right\}\right) \\
& =\phi^{n+1}\left(E_{\lambda, \mathcal{M}_{M, N}}\left(g x_{0}, f x_{0}\right)\right) \\
& \leq \phi^{n+1}\left(E_{\mathcal{M}_{M, N}}\left(g x_{0}, f x_{0}\right)\right) .
\end{aligned}
$$

Thus $E_{\lambda, \mathcal{M}_{M, N}}\left(f x_{n}, f x_{n+1}\right) \leq \phi^{n+1}\left(E_{\mathcal{M}_{M, N}}\left(g x_{0}, f x_{0}\right)\right)$ for each $\lambda \in L^{*}-\left\{0_{L^{*}}, 1_{L^{*}}\right\}$ and so

$$
E_{\mathcal{M}_{M, N}}\left(f x_{n}, f x_{n+1}\right) \leq \phi^{n+1}\left(E_{\mathcal{M}_{M, N}}\left(g x_{0}, f x_{0}\right)\right)
$$

Let $\epsilon>0$. Select $n \in\{1,2, \ldots\}$; therefore $E_{\mathcal{M}_{M, N}}\left(f x_{n}, f x_{n+1}\right)<\frac{\epsilon-\phi(\epsilon)}{K}$. For $\lambda \in L^{*}-\left\{0_{L^{*}}, 1_{L^{*}}\right\}$ there exists a $\mu \in L^{*}-\left\{0_{L^{*}}, 1_{L^{*}}\right\}$ with

$$
\begin{aligned}
E_{\lambda, \mathcal{M}_{M, N}}\left(f x_{n}, f x_{n+2}\right) & \leq K E_{\mu, \mathcal{M}_{M, N}}\left(f x_{n}, f x_{n+1}\right)+K E_{\mu, \mathcal{M}_{M, N}}\left(f x_{n+1}, f x_{n+2}\right) \\
& \leq K E_{\mu, \mathcal{M}_{M, N}}\left(f x_{n}, f x_{n+1}\right)+\phi\left(K E_{\mu, \mathcal{M}_{M, N}}\left(f x_{n}, f x_{n+1}\right)\right) \\
& \leq K E_{\mathcal{M}_{M, N}}\left(f x_{n}, f x_{n+1}\right)+\phi\left(K E_{\mathcal{M}_{M, N}}\left(f x_{n}, f x_{n+1}\right)\right) \\
& \leq K \frac{\epsilon-\phi(\epsilon)}{K}+\phi\left(K \frac{\epsilon-\phi(\epsilon)}{K}\right) \\
& \leq \epsilon .
\end{aligned}
$$

We can continue this process for every $\lambda \in L^{*}-\left\{0_{L^{*}}, 1_{L^{*}}\right\}$; then

$$
E_{\mathcal{M}_{M, N}}\left(f x_{n}, f x_{n+2}\right) \leq \epsilon
$$

For $\lambda \in L^{*}-\left\{0_{L^{*}}, 1_{L^{*}}\right\}$ there exists a $\mu \in L^{*}-\left\{0_{L^{*}}, 1_{L^{*}}\right\}$ with

$$
\begin{aligned}
E_{\lambda, \mathcal{M}_{M, N}}\left(f x_{n}, x_{n+3}\right) & \leq K E_{\mu, \mathcal{M}_{M, N}}\left(f x_{n}, f x_{n+1}\right)+K E_{\mu, \mathcal{M}_{M, N}}\left(f x_{n+1}, f x_{n+3}\right) \\
& \leq K E_{\mu, \mathcal{M}_{M, N}}\left(f x_{n}, f x_{n+1}\right)+\phi\left(K E_{\mu, \mathcal{M}_{M, N}}\left(f x_{n}, f x_{n+2}\right)\right) \\
& \leq K E_{\mathcal{M}_{M, N}}\left(f x_{n}, f x_{n+1}\right)+\phi\left(K E_{\mathcal{M}_{M, N}}\left(f x_{n}, f x_{n+2}\right)\right) \\
& \leq \epsilon-\phi(\epsilon)+\phi(\epsilon)=\epsilon
\end{aligned}
$$

from $\mathcal{M}_{M, N}\left(f x_{n+1}, f x_{n+3}, \phi(t)\right) \geq_{L} \mathcal{M}_{M, N}\left(g x_{n+1}, g x_{n+3}, t\right)=\mathcal{M}_{M, N}\left(f x_{n}, f x_{n+2}, t\right)$ we have $E_{\lambda, \mathcal{M}_{M, N}}\left(f x_{n+1}, f x_{n+3}\right) \leq \phi\left(E_{\mu, \mathcal{M}_{M, N}}\left(f x_{n}, f x_{n+2}\right)\right)$, which implies that

$$
E_{\mathcal{M}_{M, N}}\left(f x_{n}, f x_{n+3}\right) \leq \epsilon
$$

By using induction

$$
E_{\mathcal{M}_{M, N}}\left(f x_{n}, f x_{n+k}\right) \leq \epsilon \quad \text { for } k \in\{1,2, \ldots\}
$$


and we conclude that $\left\{x_{n}\right\}_{n}$ is a Cauchy sequence and by the completeness of $X,\left\{f x_{n}\right\}_{n}$ converges to a point named $z$ in $X$. Also $\left\{g x_{n}\right\}_{n}$ converges to $z$. Now we assume that the mapping $f$ is continuous. Then $\lim _{n} f f x_{n}=f z$ and $\lim _{n} f g x_{n}=f z$. Also, since $f$ and $g$ are weakly commuting,

$$
\mathcal{M}_{M, N}\left(f g x_{n}, g f x_{n}, t\right) \geq_{L} \mathcal{M}_{M, N}\left(f x_{n}, g x_{n}, t\right)
$$

Take $n \rightarrow \infty$ in the above inequality and we get $\lim _{n} g f x_{n}=f z$, by the continuity of $\mathcal{M}$. Now, we show that $z=f z$. Assume that $z \neq f z$. From (c) for each $t>0$ we have

$$
\mathcal{M}_{M, N}\left(f x_{n}, f x_{n}, \phi^{k+1}(t)\right) \geq_{L} \mathcal{M}_{M, N}\left(g x_{n}, g f x_{n}, \phi^{k}(t)\right), \quad k \in \mathbb{N}
$$

Suppose that $n \rightarrow \infty$ in the above inequality; we get

$$
\mathcal{M}_{M, N}\left(z, f z, \phi^{k+1}(t)\right) \geq_{L} \mathcal{M}_{M, N}\left(z, f z, \phi^{k}(t)\right) .
$$

Furthermore we have

$$
\mathcal{M}_{M, N}\left(z, f z, \phi^{k}(t)\right) \geq_{L} \mathcal{M}_{M, N}\left(z, f z, \phi^{k-1}(t)\right)
$$

and

$$
\mathcal{M}_{M, N}(z, f z, \phi(t)) \geq_{L} \mathcal{M}_{M, N}(z, f z, t)
$$

Also

$$
\mathcal{M}_{M, N}\left(z, f z, \phi^{k+1}(t)\right) \geq_{L} \mathcal{M}_{M, N}(z, f z, t) .
$$

Next, we have (see Remark 2.2)

$$
\mathcal{M}_{M, N}\left(z, f z, \phi^{k+1}(t)\right) \leq_{L} \mathcal{M}_{M, N}(z, f z, t) .
$$

Then $\mathcal{M}_{M, N}(z, f z, t)=C$ and from Lemma 2.3, we conclude that $z=f z$. By assumption we have $f(X) \subseteq g(X)$; then there exists a $z_{1}$ in $X$ such that $z=f z=g z_{1}$. Now,

$$
\mathcal{M}_{M, N}\left(f f x_{n}, f z_{1}, t\right) \geq_{L} \mathcal{M}_{M, N}\left(g f x_{n}, g z_{1}, \phi^{-1}(t)\right) .
$$

Take $n \rightarrow \infty$; we get

$$
\mathcal{M}_{M, N}\left(f z, f z_{1}, t\right) \geq_{L} \mathcal{M}_{M, N}\left(f z, g z_{1}, \phi^{-1}(t)\right)=1_{L^{*}},
$$

then $f z=f z_{1}$, i.e., $z=f z=f z_{1}=g z_{1}$. Also for each $t>0$ we get

$$
\mathcal{M}_{M, N}(f z, g z, t)=\mathcal{M}_{M, N}\left(f g z_{1}, g f z_{1}, t\right) \geq_{L} \mathcal{M}_{M, N}\left(f z_{1}, g z_{1}, t\right)=\varepsilon_{0}(t)
$$

since $f$ and $g$ are weakly commuting, from which we can conclude that $f z=g z$. This implies that $z$ is a common fixed point of $f$ and $g$. 
Now we prove the uniqueness. Assume that $z^{\prime} \neq z$ is another common fixed point of $f$ and $g$. Now, for each $t>0$ and $n \in \mathbb{N}$, we have

$$
\mathcal{M}_{M, N}\left(z, z^{\prime}, \phi^{n+1}(t)\right)=\mathcal{M}_{M, N}\left(f z, f z^{\prime}, \phi^{n+1}(t)\right) \geq_{L} F_{g z, g z^{\prime}}\left(\phi^{n}(t)\right)=F_{z, z^{\prime}}\left(\phi^{n}(t)\right) .
$$

Also of course we have

$$
\mathcal{M}_{M, N}\left(z, z^{\prime}, \phi^{n}(t)\right) \geq_{L} \mathcal{M}_{M, N}\left(z, z^{\prime}, \phi^{n-1}(t)\right)
$$

and

$$
\mathcal{M}_{M, N}\left(z, z^{\prime}, \phi^{n}(t)\right) \geq_{L} \mathcal{M}_{M, N}\left(z, z^{\prime}, t\right) .
$$

As a result

$$
\mathcal{M}_{M, N}\left(z, z^{\prime}, \phi^{n+1}(t)\right) \geq_{L} \mathcal{M}_{M, N}\left(z, z^{\prime}, t\right) .
$$

On the other hand we have

$$
\mathcal{M}_{M, N}\left(z, z^{\prime}, t\right) \leq_{L} \mathcal{M}_{M, N}\left(z, z^{\prime}, \phi^{n+1}(t)\right) .
$$

Then $\mathcal{M}_{M, N}\left(z, z^{\prime}, t\right)=C$, see Lemma 2.3, implies that $z=z^{\prime}$, which is contradiction. Then $z$ is the unique common fixed point of $f$ and $g$.

\section{The existence and uniqueness of solutions for a class of integral equations}

Assume that $X=C([1,3],(-\infty, 2.1443888))$ and

$$
\mathcal{M}_{M, N}(x, y, t)= \begin{cases}0 & \text { if } t \leq 0, \\ \left(\inf _{\ell \in[1,3]} \frac{t}{t+(x(\ell)-y(\ell))^{2}}, \sup _{\ell \in[1,3]} \frac{(x(\ell)-y(\ell))^{2}}{t+(x(\ell)-y(\ell))^{2}}\right) & \text { if } t>0,\end{cases}
$$

for $x, y \in X$, then $\left(M, \mathcal{M}_{M, N}, \wedge\right)$ is a complete IFTM-space with $K=2$.

We consider the mapping $T: X \rightarrow X$ by

$$
T(x(\ell))=4+\int_{1}^{\ell}\left(x(u)-u^{2}\right) e^{1-u} d u .
$$

Put $g(x)=T(x)$ and $f(x)=T^{2}(x)$. Since $f g=g f, f$ and $g$ are (weakly) commuting. Now, for $x, y \in X$ and $t>0$,

$$
\begin{aligned}
\mathcal{M}_{M, N}(f x, f y, t) \\
=\mathcal{M}_{M, N}(T(T x(\ell)), T(T y(\ell)), t) \\
=\left(\inf _{\ell \in[1,3]} \frac{t}{t+\left|\int_{1}^{\ell}(T x(u)-T y(u)) e^{1-u} d u\right|^{2}}, \sup _{\ell \in[1,3]} \frac{\left|\int_{1}^{\ell}(T x(u)-T y(u)) e^{1-u} d u\right|^{2}}{t+\left|\int_{1}^{\ell}(T x(u)-T y(u)) e^{1-u} d u\right|^{2}}\right) \\
\geq \\
=\left(\frac{t}{t+\frac{1}{e^{4}}\left|\int_{1}^{3}(T x(u)-T y(u)) d u\right|^{2}}, \frac{\frac{1}{e^{4}}\left|\int_{1}^{3}(T x(u)-T y(u)) d u\right|^{2}}{t+\frac{1}{e^{4}}\left|\int_{1}^{3}(T x(u)-T y(u)) d u\right|^{2}}\right) \\
=\mathcal{M}_{M, N}(g x, g y, t),
\end{aligned}
$$


then

$$
\mathcal{M}_{M, N}\left(f x, f y,\left(\frac{t}{e^{4}}\right) \geq_{L} \mathcal{M}_{M, N}(g x, g y, t) .\right.
$$

Thus all conditions of Theorem 2.4 are satisfied for $\phi(t)=\frac{t}{e^{4}}$ and so $f$ and $g$ have a unique common fixed point, which is the unique solution of the integral equations

$$
x(\ell)=4+\int_{1}^{\ell}\left(x(u)-u^{2}\right) e^{1-u} d u
$$

and

$$
x(\ell)=(1-\ell)^{2} e^{1-\ell}+\int_{1}^{\ell} \int_{1}^{u}\left(x(v)-v^{2}\right) e^{2-(u+v)} d v d u .
$$

\section{Competing interests}

The author declares to have no competing interests.

\section{Author's contributions}

Only the author contributed in writing this paper.

\section{Acknowledgements}

The author is grateful to the reviewers for their valuable comments and suggestions.

Received: 24 April 2016 Accepted: 13 August 2016 Published online: 24 August 2016

\section{References}

1. Park, JH: Intuitionistic fuzzy metric spaces. Chaos Solitons Fractals 22(5), 1039-1046 (2004)

2. Saadati, R, Park, JH: On the intuitionistic fuzzy topological spaces. Chaos Solitons Fractals 27(2), 331-344 (2006)

3. Saadati, R, Vaezpour, SM, Cho, YJ: Quicksort algorithm: application of a fixed point theorem in intuitionistic fuzzy quasi-metric spaces at a domain of words. J. Comput. Appl. Math. 228(1), 219-225 (2009)

4. Deschrijver, G, Kerre, EE: On the relationship between some extensions of fuzzy set theory. Fuzzy Sets Syst. 133(2), 227-235 (2003)

5. Chauhan, S, Shatanawi, W, Kumar, S, Radenovic, S: Existence and uniqueness of fixed points in modified intuitionistic fuzzy metric spaces. J. Nonlinear Sci. Appl. 7(1), 28-41 (2014)

6. Latif, A, Kadelburg, Z, Parvaneh, V, Roshan, JR: Some fixed point theorems for G-rational Geraghty contractive mappings in ordered generalized b-metric spaces. J. Nonlinear Sci. Appl. 8(6), 1212-1227 (2015)

7. Ozturk, V, Turkoglu, D: Common fixed point theorems for mappings satisfying (E.A)-property in b-metric spaces. J. Nonlinear Sci. Appl. 8(6), 1127-1133 (2015)

8. Kadelburg, Z, Radenovic, S: Pata-type common fixed point results in b-metric and b-rectangular metric spaces. J. Nonlinear Sci. Appl. 8(6), 944-954 (2015)

9. Atanassov, KT: Intuitionistic fuzzy sets. Fuzzy Sets Syst. 20(1), 87-96 (1986)

10. Khamsi, MA, Kreinovich, VY: Fixed point theorems for dissipative mappings in complete probabilistic metric spaces. Math. Jpn. 44(3), 513-520 (1996)

11. O'Regan, D, Saadati, R: Nonlinear contraction theorems in probabilistic spaces. Appl. Math. Comput. 195(1), 86-93 (2008)

12. Latif, $A$, Abbas, $M$, Hussain, $A$ : Coincidence best proximity point of $F_{g}$-weak contractive mappings in partially ordered metric spaces. J. Nonlinear Sci. Appl. 9(5), 2448-2457 (2016)

13. Hussain, N, Khaleghizadeh, S, Salimi, $P$, Abdou, AAN: A new approach to fixed point results in triangular intuitionistic fuzzy metric spaces. Abstr. Appl. Anal. 2014, Article ID 690139 (2014)

14. Hussain, N, Isik, H, Abbas, M: Common fixed point results of generalized almost rational contraction mappings with an application. J. Nonlinear Sci. Appl. 9(5), 2273-2288 (2016)

15. Hussain, N, Arshad, M, Abbas, M, Hussain, A: Generalized dynamic process for generalized (f,L)-almost F-contraction with applications. J. Nonlinear Sci. Appl. 9(4), 1702-1715 (2016)

16. Hussain, N, Ahmad, J, Azam, A: On Suzuki-Wardowski type fixed point theorems. J. Nonlinear Sci. Appl. 8(6), 1095-1111 (2015)

17. Wang, S: Common fixed point theorems for weakly compatible mappings in fuzzy metric spaces using the CLRg property. J. Nonlinear Sci. Appl. 9(3), 1043-1051 (2016)

18. Tian, J-F, Hu, X-M, Zhao, H-S: Common tripled fixed point theorem for $W$-compatible mappings in fuzzy metric spaces. J. Nonlinear Sci. Appl. 9(3), 806-818 (2016)

19. Sangurlu, M, Turkoglu, D: Fixed point theorems for $(\psi \circ \varphi)$-contractions in a fuzzy metric spaces. J. Nonlinear Sci. Appl. 8(5), 687-694 (2015) 